\title{
Evolutionary Optimization of complex Systems in Uncertain Environments
}

\author{
Yaochu Jin
}

University of Surrey, UK

\begin{abstract}
This talk aims to discuss the main challenges in evolutionary optimization of complex systems to bridge the gap between the academic research and the urgent demands from industry. We will show that, while solving "hard" problems, such as multi-modal and strongly correlated problems, multi-objective optimization problems with a large number of objectives, and multi-objective optimization problems having a very complex Pareto front are popular in academia, industry is more concerned with complexity in formulating the optimization problems, choosing the right decision variables, defining the most important objectives, dealing with computationally very expensive and uncertain problems, and handling different time/cost constraints in the conceptual, design and verification phases. We will also point out that some assumptions in the present research on multi-objective and dynamic optimization are unrealistic, leading to results of little practical value.
\end{abstract}

In the presentation, several application examples from industry, such as design optimization of vehicles, high-lift wing systems, micro heat exchangers, and steel-making and continuous casting will be used to illustrate the real-world challenges in evolutionary optimization. 